\title{
Erratum to: The Combination of Surgery and Imatinib in GIST: A Reality for Localized Tumors at High Risk, an Open Issue for Metastatic Ones
}

\author{
Alessandro Gronchi, MD ${ }^{1}$ and Chandrajit P. Raut, MD, Msc ${ }^{2,3,4}$ \\ ${ }^{1}$ Department of Surgery, Sarcoma Service, Fondazione IRCCS Istituto Nazionale Tumori, Milan, Italy; ${ }^{2}$ Department of \\ Surgery, Brigham and Women's Hospital, Boston, MA; ${ }^{3}$ Center for Sarcoma and Bone Oncology, Dana-Farber Cancer \\ Institute, Boston, MA; ${ }^{4}$ Harvard Medical School, Boston, MA
}

\section{ERRATUM TO: ANN SURG ONCOL DOI 10.1245/S10434-011-2191-4}

In the original article, Fig. 2 was not published with the correct formatting.

The figure is reprinted here correctly.

FIG. 2 Contrast-enhanced magnetic resonance image of primary low rectal gastrointestinal stromal tumor before (right) and after (left) preoperative imatinib for 8 months. Because the tumor harbored an exon 9 mutation, the patient was treated with a higher dose. Surgery consisted of local excision rather than a formal low anterior resection

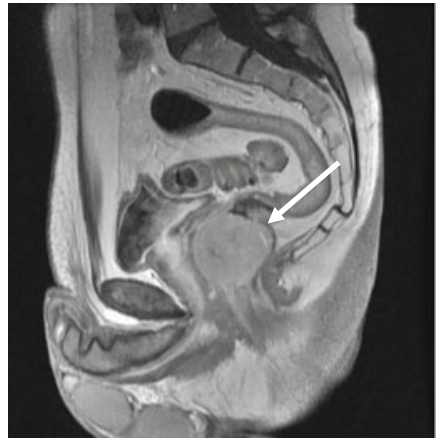

IM $800 \mathrm{mg} /$ day $x 8$ mos

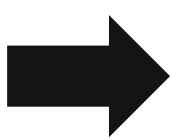

Kit exon 9 mutation

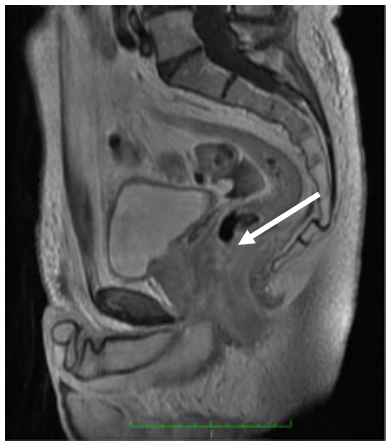

- Local excision through perineal approach
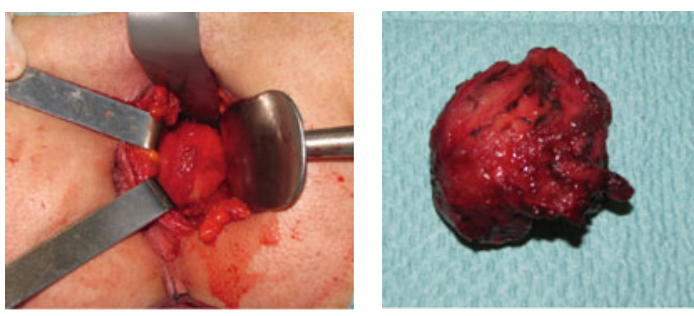

The online version of the original article can be found under doi:10.1245/s10434-011-2191-4.

(C) Society of Surgical Oncology 2012

Published Online: 1 February 2012

A. Gronchi, MD

e-mail: alessandro.gronchi@istitutotumori.mi.it 